\title{
The one-stop clinic as the standard of out-patient care in a hospital urology department
}

\author{
Alvaro Páez, Enrique Redondo, Javier Sáenz, Mercedes Marín, Nuria Juárez and Manuel Durán \\ Urology Department, Hospital Universitario de Fuenlabrada, Madrid, Spain, Universidad Rey \\ Juan Carlos, Madrid, Spain
}

\begin{abstract}
Purpose: To evaluate the performance of a 'one-stop' clinic in terms of proportion of discharges or inclusion in surgical waiting lists.

Materials and Methods: All patients were referred from primary care facilities (population 220.646) and from different departments in the hospital. Eight senior urologists, two registered nurses and two nurse attendants participated in the experience. Prior to the start of the project, referral protocols had been agreed with the primary care physicians involved. Compliance with the protocols was periodically tested. Eventually 5537 first visits (January-December 2009) where evaluable.

Results: Overall, the 'one-stop' format proved feasible in $74.2 \%$ of the patients (4108/5537). Patients, who successfully used the 'one-stop' format, were significantly younger than those who required additional consultations (43 vs 50 years old, respectively, Student's $t$ test $<0.001$ ).

For obvious reasons the 'one-stop' format was universally possible in male sterilization and penile phimosis patients. Similarly, the 'one-stop' policy was applied in most consultations due to male sexual dysfunction $(75 \%)$ and urinary tract infection (73\%). Other health problems, such as haematuria (62\%) and renal colic (46\%), required more than one visit so that care of the patient reverted to the traditional, outpatient care model.

Conclusion: A 'one-stop' philosophy is feasible for a number of procedures in a urological outpatient clinic. The costs to implement such an approach would be limited to managerial expenditure.
\end{abstract}

Key words: day care; outpatients; primary health care; urology; clinic visit

Int Braz J Urol. 2011; 37: 623-629

\section{INTRODUCTION}

The Spanish National Health System (NHS) suffers an enormous stress. Average number of visits in 2008 was 9 per inhabitant, resulting in more than 400 million consultations (1). Such cyclopean duty is inevitably full of inefficiency: under the existing system it is not unusual that patients need to wait weeks for an appointment with their specialist. After the initial out-patient appointment, several visits are generally necessary prior to a management decision, as it is a standard procedure in Spain to perform diagnostic imaging and tests by appointment. Fortunately many urology departments have now their own endoscopy units and imaging facilities (echography). Nevertheless, follow-up visits are usually scheduled after the examinations have been carried out to discuss the diagnosis. The vast majority of patients need no further follow-up, but some enter into specific clinics for variable periods of time. To overcome these difficulties, the organizational reaction is to increase the size of the clinics, but in turn this response is likely, by weight of patient numbers, to decrease patient accessibility rather than increase it (2).

Nevertheless, accessibility is only a part of the problem; the current set-up lacks flexibility and creativity. An example would be the treatment 
of well-defined urological problems, such as haematuria or scrotal swelling, which tend to require multiple visits (often 2 or 3 , sometimes more) to the traditional urology outpatient clinic. During these visits, the patient will meet several different physicians, each having their own view of the patient's case. During this period of time, the patient must cope with his anxieties and fears until a final clinical decision can be made.

However, although improving patient accessibility and creating specific 'one-stop' clinics might be the answer for certain groups of patients (e.g. oncology cases), it can be argued that one-stop clinics have the potential to result in inequity, while not making sufficient improvement beyond that received by patients in the traditional model of specialized care.

The aim of our 'one-stop' programme was to provide all the services needed for patients referred to our facilities at a single visit.

The present study shows the results of a 'onestop' clinic for all patients referred to the urology department of a 400-bed public hospital during 2009.

\section{MATERIALS AND METHODS}

We used the percentage of patients diagnosed and discharged, or diagnosed and included in the surgical waiting list (when indicated), as an indicator of the efficacy of the 'one-stop' approach.

There was not a formal 'one-stop' clinical setting or was the clinical setting specially designated. Simply, all patients referred to our facilities for a first consultation (irrespectively of their age, gender or reason for consultation) were processed using the 'one-stop' approach.

Patients were referred to our urological department from public primary care facilities (ten different public primary care centres, general population 220,646) and from other clinical departments in the Hospital Universitario de Fuenlabrada, Madrid, Spain. Three daily outpatient clinics were made available for these patients.

For patients referred from primary care, general practitioners (GP) were able to book patients directly into the 'one-stop' clinic. There were no formal restrictions for referrals. However, the Spanish
NHS is not an open-access system, and GPs were expected to act as gate-keepers, i.e. to make clinical decisions about the suitability of patients for referral to secondary urological care.

For the study, referral criteria were agreed (3) and were periodically tested (4). Because the 'one-stop' approach was an initiative of the urology department, and customers (primary care and clinical departments in the hospital) have a passive role, there were no financial charges.

At the 'one-stop' clinic, the patient's basic clinical information could be accessed on-line. Once in our facilities, state-of-the-art diagnostic procedures where provided when needed.

Cases that finally resulted in inclusion in the surgical waiting list (WL) or discharge after a single visit were considered as managed under the 'onestop' format. All other patients (i.e. patients needing another visit to the hospital for additional tests and/ or urological work-up) were considered as managed under the traditional 'standard care' format.

Eight urologists, two registered nurses and two nurse attendants participated in the study. Registered nurses had previously been trained in basic urological echography, therapeutic endoscopy (pigtail removal) and urodynamics; they also had been given permission to conduct a minor surgery clinic (penile phimosis and male sterilization).

Ultrasound and endoscopy units were permanently operative and elemental urinalysis and plain abdominal films (KUB) were immediately available. Some minor surgical procedures (e.g. wound care, abscess drainage), uroflowmetry, urethral catheterization and urethral dilation were available in the outpatient clinic. However, non-conventional imaging and blood tests were not permanently available. Similarly, more elaborate minor surgical procedures, e.g. ultrasound-guided percutaneous nephrostomy, were not allowed in the 'one-stop' clinics.

Eventually, every urologist and nurse committed to achieving a minimum of $73 \%$ 'one-stop' cases. Doctors and nurses developed their own clinical responsibility and decided themselves upon the applicability of the 'one-stop' philosophy to the patient presenting to them. In general, when it was overwhelmingly clear that the 'one-stop' could not be supported (especially in cases requiring blood tests 
or further imaging or pathology), a shift towards the standard approach was then decided. Thus, all patients were considered for the 'one-stop' approach until it became clear that a 'one-stop' approach was not sufficient and care of the patient then reverted to the traditional, standard approach.

For the present study, a total of 5537 first visits were considered. To approach the programme performance, the relationship between the consultation outcome ('one-stop' or 'standard care') and patient's age, gender, and reason for consultation was tested. More than 200 different reasons for consultation were recorded; for the purposes of conducting a reasonable statistical analysis, the reasons were summarized into 15 major arbitrary categories.

To decide about the overall programme efficacy, the number of revisits after discharge was considered.

Data were treated using the commercially available statistical program SPSS v11.5. The chi $^{2}$ and Student's t tests were used when appropriate. A $95 \%$ statistical significance was considered for all comparisons.

\section{RESULTS}

The average age of the patients $(79.5 \%$ males) was 45 years (SD 18.7, range 1 month to 96 years). Table-1 shows the reasons for consultation grouped into the 15 major clinical syndromes. Overall, the 'one-stop' was achieved in $74.2 \%$ of the cases (4108/5537).

Eventually, it was observed that patients who were best able to take advantage of the 'onestop' format were significantly younger than those managed under the 'standard' format (43 and 51 years, respectively, Student's t test $<0.001)$. A clear relationship between age group and the percentage of patients served with the 'one-stop' format was evident $\left(\mathrm{chi}^{2}<0.001\right)$, with $87 \%$ of patients younger than 15 years being managed under this format compared with only $62 \%$ of patients aged $66-75$ years (Table-2).

The 'one-stop' format was achieved in a significantly higher percentage of men (75.8 and 67.9\% of men and women, respectively, $\left.\mathrm{chi}^{2}<0.001\right)$. Very significant differences $\left(\mathrm{chi}^{2}<0.001\right)$ were detected in the proportion of patients eventually served under the 'one-stop' format, according to the reason for consultation. For example, this was obviously going to be achievable with every case of penile phimosis and male sterilization referred to the clinic.

Similarly, the 'one-stop' approach was possible in the vast majority of consultations due to benign testicular conditions (82\%), male sexual dysfunction $(75 \%)$, urinary tract infection $(73 \%)$ and bladder filling conditions $(71 \%)$. In contrast, the 'one-stop' approach was not the rule for haematuria $(38 \%)$, and achieved in only just over half of renal colic (54\%), and genitourinary malformations (56\%) (Table-3).

In comparison with the patients served with the 'one-stop' format, the proportion of patients served under the 'standard' approach, who were revisited shortly after their discharge was significantly higher (13.6 and $22.8 \%$, respectively, chi $^{2}<$ 0.001 ). Average time to revisit was 102 days (SD 83.6 days, range 2 days to 339 days). Slight differences in time to revisit were present among patients served with the 'one-stop' format and under the 'standard' approach (95 and 113 days, respectively, Student's t test $<0.05$ ).

\section{DISCUSSION}

Achieving effective and efficient health care in overcrowded, busy clinic settings is a common target of many health care systems. The present study represents an initiative in the field of non-selective prioritization models. Previous selective experiences (mainly focused in surgical patients), while potentially beneficial, have produced adverse effects, fundamentally in terms of inequity: where and how exactly is care being denied to others in order to provide access to the selected patients (2). To provide more efficient services the number of out-patient and investigation appointments have to be reduced (5).

During the present study we focused in reducing evitable appointments. When universally applied, access improvement strategies, such as the one presented here, provide very variable returns. Our study confirmed several assumptions. Considering the asymmetry in the distribution of urological conditions between men and women, and the high prevalence 
Table 1 - Reasons for consultation grouped into major syndromes.

\begin{tabular}{|c|c|c|}
\hline Reasons for consultation & $n$ & $\%$ \\
\hline Urological cancer (or suspected cancer) & 421 & 7.6 \\
\hline Renal colic and/or urinary lithiasis & 614 & 11.1 \\
\hline $\begin{array}{l}\text { Acquired degenerative conditions of the genitourinary tract (urethral stenosis, acquired } \\
\text { obstructive uropathy, and renal cysts) }\end{array}$ & 68 & 1.2 \\
\hline Penile phimosis & 423 & 7.6 \\
\hline Male lower urinary tract symptoms & 211 & 3.8 \\
\hline Macroscopic and microscopic haematuria & 177 & 3.2 \\
\hline Urinary tract infection & 301 & 5.4 \\
\hline Disorders of bladder filling (urgency, incontinence, enuresis) & 488 & 8.8 \\
\hline Malformations of the genitourinary tract & 59 & 1.1 \\
\hline $\begin{array}{l}\text { Non-neoplastic disorders of the male genitalia (penile pain, chronic prostatitis, } \\
\text { hematospermia) }\end{array}$ & 100 & 1.8 \\
\hline Male sterilization & 483 & 8.7 \\
\hline Male sexual disorders (erectile dysfunction, premature ejaculation) and subfertility & 380 & 6.9 \\
\hline Testicular pain, varicocele, hydrocele & 385 & 7.0 \\
\hline Voiding dysfunctions unrelated to benign prostatic hypertrophy & 358 & 6.5 \\
\hline Non-classifiable conditions & 1069 & 19.3 \\
\hline Total & $\mathbf{5 5 3 7}$ & 100.0 \\
\hline
\end{tabular}

Table 2 - Relationship between age-group and type of approach ('one-stop' or standard care). In parentheses, percent.

\begin{tabular}{lcccc}
\hline \multirow{2}{*}{ Age group } & \multicolumn{3}{c}{ Approach, $\mathbf{n}(\%)$} & \multirow{2}{*}{ Square chi test } \\
\cline { 2 - 4 } & 'One-stop' & Standard care & Total & \\
\hline$<15$ & $283(87.3)$ & $41(12.7)$ & 324 & \\
$15-25$ & $397(81.0)$ & $93(19.0)$ & 490 & \\
$26-35$ & $739(78.7)$ & $200(21.3)$ & 939 & \\
$36-45$ & $821(77.8)$ & $234(22.2)$ & 1055 & \\
$46-55$ & $804(73.6)$ & $289(26.4)$ & 1093 & \\
$56-65$ & $609(67.1)$ & $299(32.9)$ & 900 & \\
$66-75$ & $253(61.3)$ & $160(38.7)$ & 413 & \\
$>75$ & $202(64.1)$ & $113(35.9)$ & 315 & \\
Total & $\mathbf{4 1 0 8 ( 7 4 . 2 )}$ & $\mathbf{1 4 2 9}(\mathbf{2 5 . 8 )}$ & $\mathbf{5 5 3 7}$ & \\
\hline
\end{tabular}

of some health problems in certain age-groups, the findings involving age and gender are not surprising: in general, the 'one-stop' approach was more frequently substantiated in males (with a high prevalence of consultations due to penile phimosis and male sterilization, both not needing extra consultations to gain a diagnosis). The 'one-stop' format was more successful in younger patients, who are usually referred for simpler consultations. Interestingly the 'one-stop' approach was progressively more dif- 
Table 3 - Relationship between clinical syndrome and type of approach ('one-stop' or standard care). In parentheses, percent.

\begin{tabular}{|c|c|c|c|c|}
\hline \multirow[t]{2}{*}{ Reasons for consultation } & \multicolumn{3}{|c|}{ Approach, n (\%) } & \multirow{2}{*}{$\begin{array}{l}\text { Square } \\
\text { chi test }\end{array}$} \\
\hline & 'One-stop' & Standard care & Total & \\
\hline Urological cancer (or suspected cancer) & $242(57.5)$ & $179(42.5)$ & 421 & \\
\hline Renal colic and/or urinary lithiasis & $331(53.9)$ & $283(46.1)$ & 614 & \\
\hline $\begin{array}{l}\text { Acquired degenerative conditions of the genitourinary } \\
\text { tract (urethral stenosis, acquired obstructive uropathy, } \\
\text { and renal cysts) }\end{array}$ & $40(58.8)$ & $28(41.2)$ & 68 & \\
\hline Penile phimosis & $420(99.3)$ & $3(0.7)$ & 423 & \\
\hline Male lower urinary tract symptoms & $145(68.7)$ & $66(31.3)$ & 211 & \\
\hline Macroscopic and microscopic hematuria & $67(37.9)$ & $110(62.1)$ & 177 & \\
\hline Urinary tract infection & $221(73.4)$ & $80(26.6)$ & 301 & \\
\hline $\begin{array}{l}\text { Disorders of bladder filling (urgency, incontinence, } \\
\text { enuresis) }\end{array}$ & $349(71.5)$ & $139(28.5)$ & 488 & $<0.001$ \\
\hline Malformations of the genitourinary tract & $33(55.9)$ & $26(44.1)$ & 59 & \\
\hline $\begin{array}{l}\text { Non-neoplastic disorders of the male genitalia (penile } \\
\text { pain, chronic prostatitis, hematospermia) }\end{array}$ & $64(64.0)$ & $36(36.0)$ & 100 & \\
\hline Male sterilization & $480(99.4)$ & $3(0.6)$ & 483 & \\
\hline $\begin{array}{l}\text { Male sexual disorders (erectile dysfunction, premature } \\
\text { ejaculation) and subfertility }\end{array}$ & $286(75.3)$ & $94(24.7)$ & 380 & \\
\hline Testicular pain, varicocele, hydrocele & $315(81.8)$ & $70(18.2)$ & 385 & \\
\hline $\begin{array}{l}\text { Voiding dysfunctions unrelated to benign prostatic } \\
\text { hypertrophy }\end{array}$ & $240(67.0)$ & $118(33.0)$ & 358 & \\
\hline Non-classifiable conditions & $875(81.9)$ & $194(18.1)$ & 1069 & \\
\hline Total & $4108(74.2)$ & $1429(25.8)$ & 5537 & \\
\hline
\end{tabular}

ficult to apply with male age; this trend with age was not found among women, perhaps due to the lack of feminine minor surgical conditions affecting younger women.

Conditions that were unlikely to require more than an empirical approach or simple diagnostic tests (i.e. echography, cystoscopy, elemental urinalysis), such as benign scrotal enlargement, male sexual dysfunction, urinary tract infection and bladder filling disorders, were mostly managed in a single visit. Similar results have been published elsewhere (6). Experiences in the field of cardiology have also resulted in excellent percentages of pa- tients diagnosed and discharged after a single visit (7). Significant improvements in waiting times and patients' satisfaction are the rule $(6,7)$. In our personal experience, $95.8 \%$ of the patients considered the 'one-stop' policy as 'adequate' or 'very adequate' in spite of the long transit times on the day of their visit (personal communication, data not published).

In summary, the need for histological and imaging studies were the main limitations for the 'one-stop' clinic. Nevertheless, this study proved that the 'one-stop' clinic can become the standard in urology. It can also be as robust as the traditional standard approach in terms of clinical effectiveness. 
Interestingly, revisits (due to the original health problem) were less frequent among patients served under the 'one-stop' approach.

It should be stressed that communication abilities are very important when applying the 'one-stop' approach. The patient will only have a single contact with the urologist, so it is essential that communication is fluent and the patient has a clear clinical understanding of what has been decided. A detailed report should be offered to every patient at discharge. Another challenge with 'onestop' clinics is the 'need' for a diagnosis: delivering a diagnosis in a matter of minutes can initially be difficult when one is not used to be profoundly operative. Professionals also need time to get used to the 'one-stop' model to feel confident and fully productive in using it.

The benefits for users seem clear: less appointments to gain a diagnosis, relationship with a single urologist, and avoiding the anxiety related to the delay of a firm diagnosis $(6,8,9)$. Another intangible social benefit of the 'one-stop' approach is increased accessibility. Just taking into account, the echographies performed during the study period (1582), and assuming that the same number of follow-up visits were saved, a minimum of 1582 second-appointments slots were vacated. Currently, our outpatient clinic receives 60 new appointments and 21 follow-up visits per day. Saving 1582 follow-up visits means shortening the waiting list for a follow-up consultation by 75 days (13-15 weeks). According to the current fee system (personal communication, data not published), second visits in our department cost 59.64 euros. A formal cost-analysis of our policy was not undertaken, but on this basis savings for the Spanish NHS could approach 95,000 euros per year. Similar services have resulted in savings for other health systems (5).

Generalization of the 'one-stop' approach needs substantial cultural changes: the traditional format for a first-visit to the specialist in which typically diagnostic tests are ordered and a new appointment is scheduled must be replaced. Similarly, terms for referral and clinical protocols need to be developed and agreed. Facilities need to be professional and patient-friendly. Diagnostic units (echography and cystoscopy) should be perma- nently operative. As a 'side effect', the outpatient area becomes a very rigid structure were there is no room for unexpected, lengthy procedures (i.e., difficult urethral catheterization using multiple catheters and followed by suprapubic bladder drainage, or percutaneous ultrasound-guided nephrostomy).

Implementing the 'one-stop' clinic philosophy can result in serious stress for a clinical department because the condensation of activities can become very important. In such scenario, the staff require great versatility. In this field, nurse empowerment is crucial. It has already been proven that nurses can be very effective in gastrointestinal endoscopy, nuclear medicine, computed tomography, echography and mammography procedures (10-15). Because of their clinical training and basic skills and abilities, they represent ideal partners for urologists in a 'one-stop' clinic setting.

Outpatient policies in the Spanish public NHS need an urgent update. Our study represents an initiative in this field. Only free-of-cost organizational changes need to be implemented to switch from the standard, tedious, approach to the more vigorous and efficient 'one-stop' model.

\section{CONCLUSIONS}

The 'one-stop' format can be substantiated in most patients and should be the rule in urology clinics. The 'one-stop' format is exigent for professionals but the benefit in terms of reduction in the number of consultations is such that the 'pain' involved in making the change really is worth it.

Partial funding for this project was provided by the Spanish Fondo de Investigación Sanitaria (FIS 97/0209)

\section{ACKNOWLEDGEMENTS}

We are indebted to Ms.Karin Plass for her generous assistance during the review process of this article.

\section{CONFLICT OF INTEREST}

None declared. 


\section{REFERENCES}

1. Sistema Nacional de Salud (The Spanish National Health System). España 2010 (Spain, 2010). Anexo D. Principales datos y cifras de la Sanidad en España (Addendum. Main data and figures of the Spanish Health System). Available from http://www.msc.es/ organizacion/sns/docs/sns2010/Anexo_04.pdf. [Last accessed Aug 21 2010].

2. Devlin N, Harrison A, Derrett S: Waiting in the NHS: part 1--a diagnosis. J R Soc Med. 2002; 95: 223-6.

3. Páez Borda A, Redondo González E, Ríos González E, Linares Quevedo A, Sáenz Medina J, Castillón Vela I: Adequacy of referral from primary care to a Department of Urology. Actas Urol Esp. 2007; 31: 1166-71.

4. Páez Borda A, Sáenz Medina J, Redondo González E, Fernández Montarroso L, Marín Valero M, López García-Franco A: An intervention to improve the adequacy of referrals from primary care to a department of urology. Actas Urol Esp. 2009; 33: 1122-8.

5. Thomas RE, Grimshaw JM, Mollison J, McClinton S, McIntosh E, Deans H, et al.: Cluster randomized trial of a guideline-based open access urological investigation service. Fam Pract. 2003; 20: 646-54.

6. Coull N, Rottenberg G, Rankin S, Pardos-Martinez M, Coker B, Jenkins E, et al.: Assessing the feasibility of a one-stop approach to diagnosis for urological patients. Ann R Coll Surg Engl. 2009; 91: 305-9.

7. Falces C, Sadurní J, Monell J, Andrea R, Ylla M, Moleiro A, et al.: One-stop outpatient cardiology clinics: 10 years' experience. Rev Esp Cardiol. 2008; 61: 530-3.

8. Zambrana JL, Jiménez-Ojeda B, Marín M, Almazán S: La consulta única o de alta resolución como una alternativa de eficiencia a las consultas externas hospitalarias tradicionales (One-stop clinic as an efficient alternative to the traditional outpatient clinics). Med Clin (Barc) 2002; 118: 302-5.

9. Queipo Zaragozá JA, Moral Baltuille A, Beltrán Meseguer JF, Marco Aznar A, Borrell Palanca A, Chicote Pérez F, et al.: Urological attendance in the Integral Health Center "Alto Palancia": model of primary-specialized integration and unique consultation. Actas Urol Esp. 2005; 29: 305-10.
10. Williams J, Russell I, Durai D, Cheung WY, Farrin A, Bloor K, et al.: Effectiveness of nurse delivered endoscopy: findings from randomised multi-institution nurse endoscopy trial (MINuET). BMJ. 2009; 338: b231.

11. Malcolm A: Medical imaging techniques: implications for nursing care. Nurs Stand. 2006; 20: 46-51.

12. Baumann BM, McCans K, Stahmer SA, Leonard MB, Shults J, Holmes WC: Caregiver and health care provider satisfaction with volumetric bladder ultrasound. Acad Emerg Med. 2007; 14: 903-7.

13. Baumann BM, McCans K, Stahmer SA, Leonard MB, Shults J, Holmes WC: Volumetric bladder ultrasound performed by trained nurses increases catheterization success in pediatric patients. Am J Emerg Med. 2008; 26: 18-23.

14. Practice Committee of the American Soceity for Reproductive Medicine: Position statement on nurses performing focused ultrasound examinations in a gynecology/infertility setting. Fertil Steril. 2006; 86(5 Suppl 1): S121.

15. Stringer M, Miesnik SR, Brown LP, Menei L, Macones GA: Limited obstetric ultrasound examinations: competency and cost. J Obstet Gynecol Neonatal Nurs. 2003; 32: 307-12.

Submitted for publication:

October 27, 2010

$\overline{\text { Accepted after revision: }}$

April 11, 2011

\section{Correspondence address:}

Dr. Alvaro Páez

Servicio de Urología, Hospital Universitario de Fuenlabrada

Camino del Molino 2,

Fuenlabrada, 28942, Madrid, Spain

Fax: + 3491 600-6186

E-mail: apaez.hflr@salud.madrid.org 